\title{
Long Noncoding RNA DLGAPI-ASI Promotes the Aggressive Behavior of Gastric Cancer by Acting as a ceRNA for microRNA-628-5p and Raising Astrocyte Elevated Gene I Expression
}

This article was published in the following Dove Press journal:

Cancer Management and Research

Jiying Deng'

Qin Zhang ${ }^{2}$

Lianwei $\mathrm{Lu}^{3}$

Chunxia Fan'

'Department of General Surgery, Gaomi People's Hospital, Gaomi, Shandong 26I500, People's Republic of China; ${ }^{2}$ Department of Neurosurgery, Gaomi People's Hospital, Gaomi, Shandong 26I500, People's Republic of China; ${ }^{3}$ Department of Radiology, Weifang People's Hospital, Weifang, Shandong 261000, People's Republic of China
Correspondence: Chunxia Fan Email fan_chunxiagaomi@163.com
Purpose: The long noncoding RNA DLGAP1 antisense RNA 1 (DLGAP1-AS1) plays welldefined roles in the malignant progression of hepatocellular carcinoma. The purpose of this study was to determine whether DLGAP1-AS1 affects the aggressive behavior of gastric cancer (GC).

Methods: DLGAP1-AS1 expression in GC tissue samples and cell lines was determined by reverse-transcription quantitative PCR. GC cell proliferation, apoptosis, migration, invasion, and tumor growth in vitro as well as in vivo were examined by the Cell Counting Kit-8 assay, flow-cytometric analysis, transwell migration and invasion assays, and xenograft model experiments, respectively.

Results: DLGAP1-AS1 was overexpressed in GC tissue samples and cell lines. Among patients with GC, the increased level of DLGAP1-AS1 correlated with tumor size, TNM stage, lymph node metastasis, distant metastasis, and shorter overall survival. The knockdown of DLGAP1-AS1 suppressed GC cell proliferation, migration, and invasion in vitro, as well as promoted cell apoptosis and hindered tumor growth in vivo. Mechanistically, DLGAP1-AS1 functioned as a competing endogenous RNA for microRNA-628-5p (miR$628-5 p)$ in GC cells, thereby increasing the expression of the miR-628-5p target astrocyte elevated gene 1 (AEG-1). Functionally, the recovery of the miR-628-5p/AEG-1 axis output attenuated the effects of DLGAP1-AS1 knockdown in GC cells.

Conclusion: DLGAP1-AS1 is a pleiotropic oncogenic lncRNA in GC. DLGAP1-AS1 plays a pivotal part in the oncogenicity of GC in vitro and in vivo by regulating the miR-628-5p/ AEG-1 axis. DLGAP1-AS1, miR-628-5p, and AEG-1 form a regulatory pathway to facilitate GC progression, suggesting this pathway as an effective target for the treatment of GC.

Keywords: DLGAP1 antisense RNA 1, microRNA 628-5p, astrocyte elevated gene 1

\section{Introduction}

Gastric cancer (GC) is the fourth most common cancer and the third major cause of cancer-associated deaths globally. ${ }^{1}$ Approximately 850,000 new GC cases and 650,000 associated deaths are registered every year. ${ }^{2}$ Currently, surgical resection followed by chemoradiation and adjuvant chemotherapy is the first-line therapeutic strategy for patients with GC. ${ }^{3}$ Tremendous advances in the diagnosis and management of GC have been made in the past several decades; unfortunately, the therapeutic efficacy of the existing modalities is still not ideal, with an overall 5-year survival rate 
of only $20 \%{ }^{4,5}$ Recurrence and metastasis are the major obstacles for the curative treatment of $\mathrm{GC}^{6}$ In addition, chemoresistance contributes to the poor therapeutic outcomes for patients with GC diagnosed at an advanced stage. ${ }^{7}$ Multiple factors, including Helicobacter pylori infection, diet, smoking, and obesity, play important roles in gastric carcinogenesis and GC progression; however, the detailed molecular events underlying GC pathogenesis are not well understood. Hence, an in-depth understanding of the mechanisms underlying GC initiation, progression, and chemoresistance is urgently needed for identifying promising diagnostic options and therapeutic interventions.

Long noncoding RNAs (lncRNAs) belong to a cluster of transcripts over 200 nucleotides in length and lacking protein-coding capacity. ${ }^{8}$ They can modulate gene expression at the epigenetic, transcriptional, and post-transcriptional levels, and these regulatory roles are carried out through various mechanisms, including interactions with RNA, proteins, and DNA. ${ }^{9-11}$ Intriguingly, lncRNAs have attracted much attention due to their significant correlations with carcinogenesis and cancer progression. ${ }^{12-14}$ An increasing number of studies have shown that numerous lncRNAs are abnormally expressed in GC. ${ }^{15-17}$ Notably, there is increasing evidence supporting a close relationship between lncRNA dysregulation and malignant characteristics in GC. ${ }^{18,19}$

MicroRNAs (miRNAs, miRs) are classified as singlestranded noncoding short RNAs approximately 19-25 nucleotides in length. ${ }^{20}$ MiRNAs serve as major posttranscriptional regulators of gene expression by directly interacting with the 3 ' untranslated regions (3'-UTRs) of their target mRNAs, which can result in the subsequent degradation of a target mRNA or suppression of its translation. $^{21}$ MiRNAs are implicated in nearly all known physiological and pathological processes, including carcinogenesis and cancer progression. ${ }^{22}$ Accordingly, comprehensive research into the involvement of lncRNA and miRNAs in GC progression may facilitate the development of promising treatment options, and thereby improve clinical outcomes among patients with this disease.

A lncRNA termed DLGAP1-AS1 performs welldefined functions in the malignant progression of hepatocellular carcinoma. ${ }^{23}$ Nonetheless, it is not known whether DLGAP1-AS1 plays a role in the regulation of GC oncogenicity. In this study, we attempted to quantify DLGAP1AS1 expression in GC and determine the clinical relevance of DLGAP1-AS1 in GC. We further aimed to investigate the role of DLGAP1-AS1 in the malignant characteristics of GC and clarify the underlying molecular events.
MiR-628-5p is weakly expressed in pancreatic ductal adenocarcinoma, ${ }^{24}$ epithelial ovarian cancer $^{25}$ and glioma, ${ }^{26}$ and inhibits the malignancy of these cancer types. On the contrary, miR-628-5p is highly expressed in osteosarcoma and promotes cancer progression. ${ }^{27}$ AEG-1 is upregulated in GC, which is correlated with adverse clinical features and poor prognosis. ${ }^{28-30}$ Functionally, AEG-1 performes cancer-promoting actions in gastric carcinogenesis and cancer progression, and is involved in multiple aggressive phenotype. ${ }^{31-35}$ Yet, as far as we know, there has been no study that has explored the issue of DLGAP1-AS1, miR628-5p, and AEG-1 in GC. Herein, we also attempted to address the functions and associations between DLGAP1AS1, miR-628-5p, and AEG-1 in GC.

\section{Materials and Methods Tissue Samples and Cell Lines}

Sixty-three pairs of samples of tumor tissues and the corresponding adjacent non-tumor tissues were collected from patients with GC at Gaomi People's Hospital. All these patients underwent surgical resection and had not been treated with chemotherapy, radiotherapy, or other anticancer modalities. The experimental protocols of our current study were approved by the Ethics Committee of Gaomi People's Hospital and were performed in accordance with the Declaration of Helsinki. In addition, all participants provided written informed consent prior to surgical resection. GC patients were followed-up, ranging for 60 months. All tissue samples were snap-frozen in liquid nitrogen after collection and then transferred to $\mathrm{a}-80^{\circ} \mathrm{C}$ cryogenic freezer.

Five human GC cell lines, MKN-45, HGC27, SNU-1, AGS, and MGC-803, were purchased from the Type Culture Collection of the Chinese Academy of Sciences (Shanghai, China). A human gastric epithelial cell line, GES-1, was obtained from American Type Culture Collection (Manassas, VA, USA). Dulbecco's modified Eagle's medium (DMEM; Gibco, Thermo Fisher Scientific, Inc., Waltham, MA, USA) containing 10\% of fetal bovine serum (FBS; Gibco, Thermo Fisher Scientific, Inc.), $100 \mathrm{U} / \mathrm{mL}$ penicillin, and $100 \mu \mathrm{g} / \mathrm{mL}$ streptomycin was utilized for cell culture. The cells were grown at $37^{\circ} \mathrm{C}$ in a humidified incubator supplied with $5 \%$ of $\mathrm{CO}_{2}$.

\section{Transient Transfection}

Oligonucleotides, including miR-628-5p mimic, negative control miRNA mimic (miR-NC), miR-628-5p inhibitor (in-miR-628-5p), and negative control miRNA inhibitor 
(in-miR-NC), were chemically synthesized by Shanghai GenePharma Co., Ltd. (Shanghai, China). The small interfering RNA (siRNA) for silencing DLGAP1-AS1 expression (si-DLGAP1-AS1), negative control siRNA (si-NC), AEG-1 overexpression plasmid (pcDNA3.1-AEG-1) containing the whole coding sequence of AEG-1 (but lacking the normal 3'-UTR), and the empty pcDNA3.1 vector were acquired from Guangzhou RiboBio Co., Ltd. (Guangzhou, China). Cells were seeded in 6-well plates at an initial density of $5 \times 10^{6}$ cells/well, followed by transfection with the abovementioned oligonucleotides or plasmids using Lipofectamine $^{\circledR} 2000$ Reagent (Invitrogen; Carlsbad, CA, USA).

\section{Reverse-Transcription Quantitative PCR (RT-qPCR)}

TRIzol Reagent (Invitrogen, Thermo Fisher Scientific, Inc.) was used for total RNA extraction from tissue samples or cells. After the extraction, the quantity and purity of total RNA were determined on a NanoDrop spectrophotometer (ND-1000; Nanodrop Technologies, Thermo Fisher Scientific, Inc.).

To quantify DLGAP1-AS1 and $A E G-1$ mRNA expression, total RNA was reverse-transcribed into cDNA using a PrimeScript RT Reagent Kit (Takara Biotechnology Co., Ltd., Dalian, China). qPCR was next carried out with SYBR Premix Ex Taq ${ }^{\mathrm{TM}}$ (Takara Biotechnology Co., Ltd., Dalian, China) on a Light Cycler 480 II Real-Time PCR system (Roche Diagnostics, Basel, Switzerland). DLGAP1-AS1 and $A E G-1$ mRNA expression were normalized to glyceraldehyde phosphate dehydrogenase $(G A P D H)$ expression.

To measure miR-628-5p expression, cDNA synthesis was carried out using a miRcute Plus miRNA First-Strand cDNA Synthesis Kit, and the synthesized cDNA was then subjected to PCR amplification using the miRcute Plus miRNA SYBR Green qPCR Kit (both form Tiangen Biotech Co., Ltd., Beijing, China). U6 small nuclear RNA acted as the control for miR-628-5p. All gene expression levels were calculated using the $2^{-\Delta \Delta C q}$ method.

\section{Subcellular Fraction Extraction}

About $1 \times 10^{7}$ cells were harvested and used for separating nuclear and cytoplasmic RNA by means of a Cytoplasmic and Nuclear RNA Purification Kit (Norgen, Ontario, Canada). The nuclear and cytoplasmic fractions were analyzed using RT-qPCR to determine the distribution of DLGAP1-AS1 expression in GC cells. GAPDH and U6 served as the cytoplasmic and nuclear controls, respectively.

\section{Cell Counting Kit-8 (CCK-8) Assay}

Transfected cells were collected at $24 \mathrm{~h}$ post-transfection and resuspended in the culture medium. Hundred microliters of the cell suspension, containing an estimated 2,000 cells, was inoculated into wells of 96-well plates. Six replicate wells were set for each group. The CCK-8 assay was performed to analyze cellular proliferation at four time points: $0,24,48$, and $72 \mathrm{~h}$ after inoculation. At every time point, $10 \mu \mathrm{L}$ of the CCK8 solution (Dojindo, Kumamoto, Japan) was added into each well prior to incubation at $37^{\circ} \mathrm{C}$ with $5 \% \quad \mathrm{CO}_{2}$ for an additional 2 h. The absorbance was read at a $450 \mathrm{~nm}$ wavelength on the spectrophotometer. Growth curves were drawn accordingly.

\section{Flow-Cytometric Analysis of Apoptosis}

Transfected cells were collected after $48 \mathrm{~h}$ of incubation, washed twice with ice-cold phosphate-buffered saline, and then used for measurement of the apoptosis rate using the Annexin V-Fluorescein Isothiocyanate (FITC) Apoptosis Detection Kit (BioLegend, Inc., San Diego, CA, USA). The transfected cells were resuspended in $1 \times$ binding buffer and transferred to a $5 \mathrm{~mL}$ culture tube, followed by incubation with $5 \mu \mathrm{L}$ of Annexin V-FITC and $5 \mu \mathrm{L}$ of the propidium iodide solution provided with the kit. Following 15 min incubation at room temperature in darkness, the proportion of apoptotic cells was measured on a flow cytometer (BD Biosciences, Franklin Lakes, NJ, USA).

\section{Transwell Migration and Invasion Assays}

Twenty-four-well transwell chambers $(8 \mu \mathrm{m}$ pore size; BD Biosciences, Franklin Lakes, NJ, USA) were used to determine the migratory and invasive abilities of the cells. For the migration assays, $5 \times 10^{4}$ transfected cells resuspended in FBS-free DMEM were seeded in the upper compartments. For the invasion assay, the chambers were precoated with Matrigel (BD Biosciences, Franklin Lakes, NJ, USA) prior to cell seeding. The upper compartments were loaded with the same number of cells as that used in the migration assay. For both assays, DMEM containing $20 \%$ of FBS was employed as a chemoattractant in the lower compartments. Transfected cells were incubated at 
$37^{\circ} \mathrm{C}$ in a humidified incubator with $5 \%$ of $\mathrm{CO}_{2}$. After 24 $\mathrm{h}$, the cells that passed through the pores in the membrane were fixed with $4 \%$ polyformaldehyde and stained with $0.5 \%$ crystal violet. After extensive washes, images were captured using a light microscope (200× magnification; Olympus Corporation, Tokyo, Japan). Six fields of view were randomly chosen, and the average cell number was determined.

\section{Xenograft Model Experiment}

Lentiviral vectors carrying DLGAP1-AS1 short hairpin RNA (shRNA; sh-DLGAP1-AS1) and negative control shRNA (sh-NC) were generated by Shanghai GenePharma Co., Ltd. AGS cells growing in the logarithmic growth phase were collected and seeded into 6-well plates. To obtain cells with stable DLGAP1-AS1 silencing, AGS cells were transfected with lentiviral vectors carrying sh-DLGAP1-AS1 or sh-NC and were then selected by incubation with puromycin.

The animal experiments were approved by the Animal Ethical Committee of Gaomi People's Hospital. All experimental steps were performed in accordance with the Animal Protection Law of the People's Republic of China-2009 for experimental animals. Female BALB/c nude mice (weighing 19-21 g, aged 5-7 weeks) were bought from Shanghai Lingchang Biotech Co., Ltd. (Shanghai, China) and were maintained under specific pathogen-free conditions. AGS cells stably transfected with either sh-DLGAP1-AS1 or sh-NC were harvested and injected into the flank of nude mice through subcutaneous inoculation. Starting at 2 weeks after inoculation, the length and width of the growing tumor xenografts were measured every 4 days using calipers. Finally, all the nude mice were euthanized by means of cervical dislocation. The tumor xenografts were excised, photographed, and weighed. The volume of tumor xenografts was calculated via the following formula: volume $=0.5 \times$ width $^{2} \times$ length.

\section{Bioinformatics Prediction and Luciferase Reporter Assay}

TargetScan 7.1 (http://www.targetscan.org/) and starBase v3.0 (http://starbase.sysu.edu.cn/) were used for predicting the potential targets of miR-628-5p. The interaction between IncRNA and miRNA was analyzed using starBase v3.0.

The fragment of the wild-type (wt) 3'-UTR of AEG-1 predicted to interact with the miR-628-5p and mutant (Mut) AEG-1 3'-UTR was produced by Shanghai GenePharma
Co., Ltd., and inserted into the pmirGLO vector (Promega Corporation, Madison, WI, USA). The constructed luciferase reported plasmids were named Wt-AEG-1 and MutAEG-1, respectively. The same experimental procedures were applied to synthesize Wt-DLGAP1-AS1 and MutDLGAP1-AS1. Cells were seeded in 24-well plates 1 day before transfection. Cotransfection of either the miR-628-5p mimic or miR-NC and either Wt or Mut reporter plasmids was performed using the Lipofectamine ${ }^{\circledR} 2000$ Reagent. Finally, the transfected cells were collected at $48 \mathrm{~h}$ posttransfection, and luciferase activity was analyzed using a Dual-Luciferase Reporter Assay System (Promega Corporation, Madison, WI, USA). Firefly luciferase activity was normalized to Renilla luciferase activity.

\section{RNA Immunoprecipitation (RIP) Assay}

The RIP assay was carried out using a Magna RIPTM RNA-Binding Protein Immunoprecipitation Kit (Millipore; Bedford, MA, USA) to evaluate the interaction between DLGAP1-AS1 and miR-628-5p in GC cells. GC cells were lysed in pre-cooled complete RIP lysis buffer, and the cell lysate was incubated with magnetic beads conjugated with human anti-Argonaute 2 (AGO2) or control anti-immunoglobulin $\mathrm{G}(\mathrm{IgG})$ antibody. Subsequent to proteinase $\mathrm{K}$ treatment, the enrichment of DLGAP1-AS1 and miR-628-5p by AGO2 was examined via RT-qPCR.

\section{Western Blot Analysis}

A radioimmunoprecipitation assay (RIPA) kit containing proteinase inhibitors (Beyotime Institute of Biotechnology, Haimen, China) was utilized for total-protein isolation from cells. The concentration of isolated total protein was assessed using a Bicinchoninic Acid Kit (Beyotime Institute of Biotechnology, Haimen, China). Equivalent amounts of protein were loaded onto each lane and separated by SDS-PAGE in a $10 \%$ gel, followed by transfer onto polyvinylidene fluoride membranes. After blocking with 5\% defatted milk powder diluted in Tris-buffered saline containing $0.5 \%$ Tween 20 (TBST), the membranes were incubated overnight at $4{ }^{\circ} \mathrm{C}$ with a primary antibody against AEG-1 (cat. No. ab124789, 1:500 dilution in TBST; Abcam, Cambridge, UK) or GAPDH (cat. No. ab128915, 1:500 dilution; Abcam). After three washes with TBST, a goat anti-rabbit horseradish peroxidaseconjugated secondary antibody (cat. No. ab205718, 1:5000 dilution; Abcam) was added and incubated at room temperature for $2 \mathrm{~h}$. Enhanced Chemiluminescence Reagent (Bio-Rad Laboratories, Hercules, CA, USA) was employed to measure the protein signals. 


\section{Statistical Analysis}

All the results are presented as the mean \pm standard deviation from at least three independent experiments. The relationship between DLGAP1-AS1 and the clinical features of patients with GC were evaluated using the $\chi^{2}$ test. Comparison of the differences between two groups was carried out using Student's $t$-test. One-way analysis of variance followed by Tukey's post hoc test was conducted to examine differences among multiple groups. The expression correlation between DLGAP1-AS1 and miR-628-5p was tested via Spearman correlation analysis. The Kaplan-Meier method was utilized to plot survival curves, followed by the log rank test to compare survival outcomes. All the data were analyzed using the SPSS 20.0 statistical software (SPSS, Inc., Chicago, IL, USA), and differences were considered statistically significant when the $\mathrm{P}$ value was less than 0.05 .

\section{Results}

\section{DLGAPI-ASI Is Upregulated in GC}

To characterize the expression profile of DLGAP1-AS1 in GC, 63 pairs of GC tissue samples and corresponding adjacent non-tumor tissues were collected, and DLGAP1AS1 expression was determined via RT-qPCR. DLGAP1AS1 was highly expressed in GC tissue samples compared with the corresponding adjacent non-tumor tissue samples (Figure 1A). Furthermore, analysis of DLGAP1-AS1 expression in GC cell lines (MKN-45, HGC27, SNU-1, AGS, and MGC-803) and the human gastric epithelial cell line GES-1 was performed through RT-qPCR. DLGAP1-
AS1 was upregulated in all five GC cell lines relative to the normal cell line GES-1 (Figure 1B).

The correlation between DLGAP1-AS1 expression and the clinical characteristics of patients with GC was elucidated in detail. All patients with GC were subdivided into either DLGAP1-AS1 low or DLGAP1-AS1 high expression groups based on the DLGAP1-AS1 median expression level among the GC tissue samples. This analysis revealed that the expression of DLGAP1-AS1 significantly correlated with tumor size $(\mathrm{P}=0.023)$, TNM stage $(\mathrm{P}=0.011)$, lymph node metastasis $(\mathrm{P}$ $=0.017)$, and distant metastasis $(P=0.027)$ among the patients with GC (Table 1). Kaplan-Meier survival curve analysis indicated that patients with GC and high DLGAP1AS1 expression had significantly shorter overall survival compared to patients with low DLGAP1-AS1 expression (Figure $1 C ; \mathrm{P}=0.032$ ). These results suggest that DLGAP1AS1 may be involved in the progression of GC.

\section{Silencing DLGAPI-ASI Expression Inhibits GC Cell Proliferation, Migration, and Invasion and Promotes Cell Apoptosis}

The GC cell lines SNU-1 and AGS demonstrated relatively higher DLGAP1-AS1 expression compared with the other three GC cell lines; hence, for our following experiments, these two cell lines were selected as the models to investigate the role of DLGAP1-AS1 in the malignancy of GC. si-DLGAP1-AS1 was transfected into SNU-1 and AGS cells to decrease endogenous DLGAP1-AS1
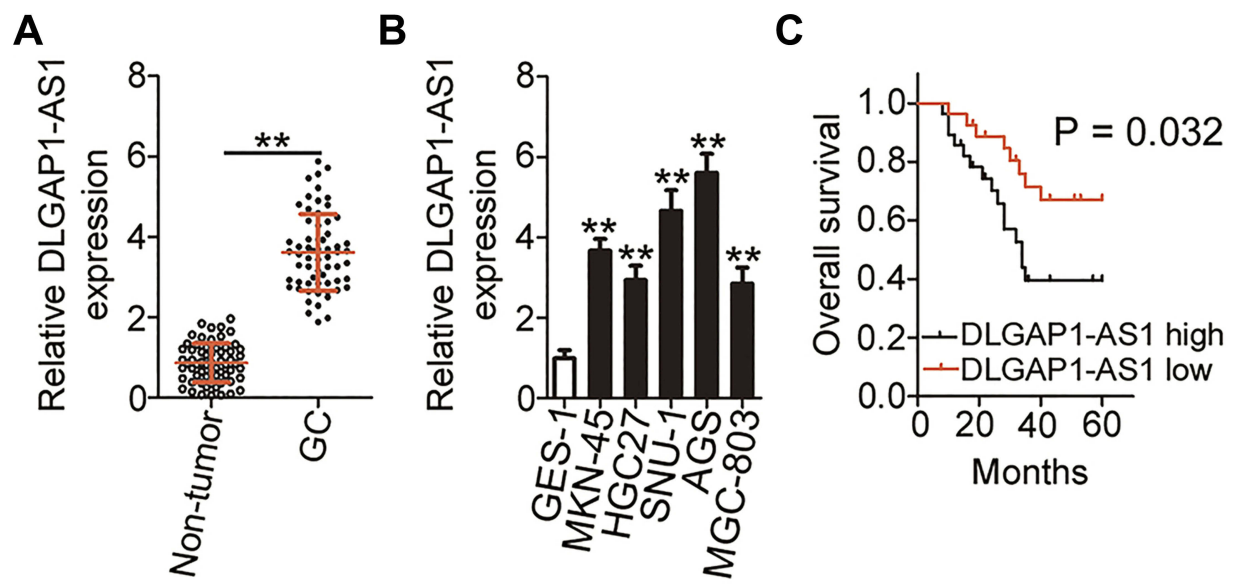

Figure I DLGAPI-ASI is overexpressed in gastric cancer (GC) tumors and cell lines. (A) DLGAPI-ASI expression in 63 pairs of GC tissue samples and corresponding adjacent non-tumor tissue samples was determined via reverse-transcription quantitative PCR (RT-qPCR). (B) RT-qPCR was carried out to assess DLGAPI-ASI expression in GC cell lines (MKN-45, HGC27, SNU-I, AGS, and MGC-803). A human gastric epithelial cell line, GES-I, served as the control. (C) Kaplan-Meier curves in relation to DLGAPI-ASI expression in patients with GC. Patients with GC showing high DLGAPI-ASI expression manifested significantly shorter overall survival compared with patients showing low DLGAPI-ASI expression $(\mathrm{P}=0.032)$. **P $<0.01$. 
Table I The Correlation Between Clinicopathological Variables and the Expression of DLGAPI-ASI in Patients with GC

\begin{tabular}{|c|c|c|c|}
\hline \multirow[t]{2}{*}{ Characteristics } & \multicolumn{2}{|c|}{ DLGAPI-ASI Expression } & \multirow[t]{2}{*}{ P-value } \\
\hline & $\begin{array}{l}\text { No. of High } \\
\text { Expression }\end{array}$ & $\begin{array}{l}\text { No. of Low } \\
\text { Expression }\end{array}$ & \\
\hline $\begin{array}{l}\text { Age (years) } \\
\quad<60 \\
\geq 60\end{array}$ & $\begin{array}{l}20 \\
12\end{array}$ & $\begin{array}{l}15 \\
16\end{array}$ & 0.315 \\
\hline $\begin{array}{l}\text { Gender } \\
\text { Male } \\
\text { Female }\end{array}$ & $\begin{array}{l}18 \\
14\end{array}$ & $\begin{array}{l}20 \\
11\end{array}$ & 0.609 \\
\hline $\begin{array}{l}\text { Tumor size } \\
\quad<3 \mathrm{~cm} \\
\geq 3 \mathrm{~cm}\end{array}$ & $\begin{array}{l}10 \\
22\end{array}$ & $\begin{array}{l}19 \\
12\end{array}$ & 0.023 \\
\hline $\begin{array}{l}\text { Differentiation } \\
\text { Well-intermediately } \\
\text { Poor }\end{array}$ & $\begin{array}{l}16 \\
16\end{array}$ & $\begin{array}{l}18 \\
13\end{array}$ & 0.616 \\
\hline $\begin{array}{l}\text { TNM stage } \\
\text { I-II } \\
\text { III-IV }\end{array}$ & $\begin{array}{l}13 \\
19\end{array}$ & $\begin{array}{l}23 \\
8\end{array}$ & 0.011 \\
\hline $\begin{array}{l}\text { Lymph node } \\
\text { metastasis } \\
\text { No } \\
\text { Yes }\end{array}$ & $\begin{array}{l}16 \\
16\end{array}$ & $\begin{array}{l}25 \\
6\end{array}$ & 0.017 \\
\hline $\begin{array}{l}\text { Distant metastasis } \\
\text { No } \\
\text { Yes }\end{array}$ & $\begin{array}{l}18 \\
14\end{array}$ & $\begin{array}{l}26 \\
5\end{array}$ & 0.027 \\
\hline
\end{tabular}

Abbreviations: DLGAPI-ASI, DLGAPI antisense RNA I; GC, gastric cancer; TNM, Tumor-node-metastasis.

expression, which was confirmed by RT-qPCR (Figure 2A). The results of the $\mathrm{CCK}-8$ assay revealed that the proliferative ability of SNU-1 and AGS cells significantly decreased following DLGAP1-AS1 downregulation (Figure 2B). Flow-cytometric analysis was performed to determine the apoptosis rate of DLGAP1-AS1-deficient SNU-1 and AGS cells. Downregulation DLGAP1-AS1 increased the apoptotic rate of SNU-1 and AGS cells (Figure 2C). We further evaluated whether DLGAP1AS1 affects the migration and invasiveness of GC cells in vitro using transwell migration and invasion assays. The knockdown of DLGAP1-AS1 impaired the migratory (Figure 2D) and invasive (Figure 2E) abilities of SNU-1 and AGS cells. These results clearly suggest that DLGAP1-AS1 plays oncogenic roles in the malignant phenotype of GC.

\section{DLGAPI-ASI Serves as a Sponge of miR-628-5p in GC Cells}

To explore the mechanisms involved in the oncogenic actions of DLGAP1-AS1, subcellular fraction extraction was performed to investigate the localization of DLGAP1-AS1 expression in GC cells. The data confirmed that DLGAP1AS1 was mostly distributed in the cytoplasm of SNU-1 and AGS cells (Figure 3A). Recent studies revealed that cytoplasmic lncRNAs act as competing endogenous RNAs (ceRNAs) to directly interact with miRNAs and reduce their expression, resulting in the upregulation of their target mRNAs. ${ }^{36-38}$ Hence, we hypothesized that DLGAP1-AS1 may work as a ceRNA in GC. Based on the results of bioinformatics prediction, miR-628-5p (Figure 3B) was selected for further evaluation due to its crucial functions in the oncogenicity of multiple human cancers. ${ }^{25-27}$

A luciferase reporter assay was carried out to confirm the targeting relationships between DLGAP1-AS1 and miR$628-5 p$ in GC cells. In SNU-1 and AGS cells, exogenous miR-628-5p expression effectively decreased the luciferase activity of Wt-DLGAP1-AS1; however, the luciferase activity of Mut-DLGAP1-AS1 was unaffected in response to miR-628-5p overexpression (Figure 3C). In addition, DLGAP1-AS1 and miR-628-5p were vastly enriched in the AGO2 antibody-treated group relative to the IgG antibodytreated group, as determined through RIP assay (Figure 3D).

RT-qPCR was performed to detect miR-628-5p in 63 pairs of GC tissue samples and corresponding adjacent non-tumor tissues. MiR-628-5p was downregulated in GC tissues compared with adjacent non-tumor tissues (Figure $3 \mathrm{E}$ ), and the expression levels of DLGAP1-AS1 and miR$628-5 \mathrm{p}$ were inversely correlated in the $63 \mathrm{GC}$ tissues (Figure 3F; $\mathrm{r}=-0.5472, \mathrm{P}<0.0001$ ). We then knocked down DLGAP1-AS1 expression in SNU-1 and AGS cells and detected the expression of miR-628-5p to further assess the interaction between DLGAP1-AS1 and miR-628-5p. Transfection with si-DLGAP1-AS1 led to a significant upregulation of miR-628-5p in SNU-1 and AGS cells (Figure $3 \mathrm{G})$. These results suggest that DLGAP1-AS1 may act as a molecular sponge for miR-628-5p in GC cells.

\section{AEG-I Is a Direct Target Gene of miR-628-5p in GC Cells and Is Positively Regulated by DLGAPI-ASI}

After verifying the downregulation of miR-628-5p in GC, we next studied the specific roles of this miRNA in GC cells. 
A

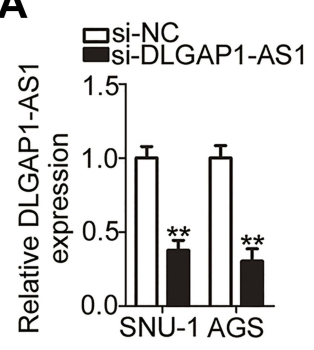

D

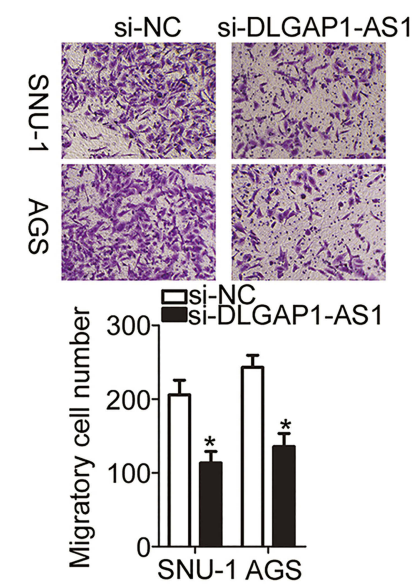

B

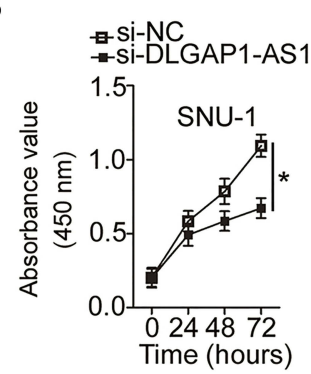

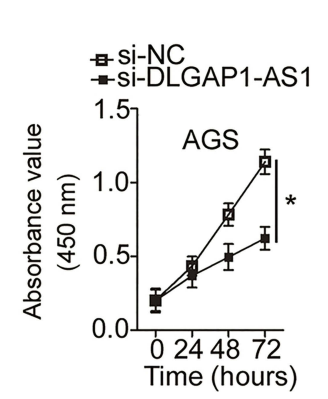

C

$\mathbf{E}$

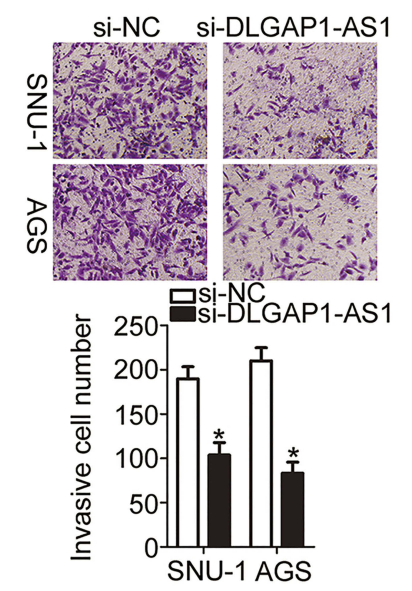

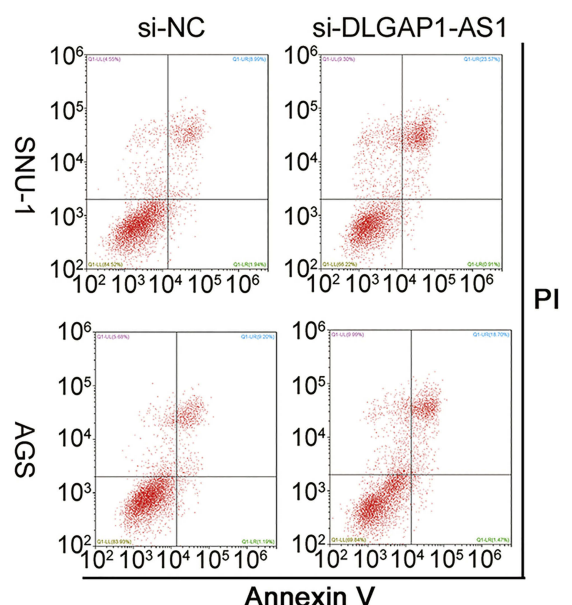

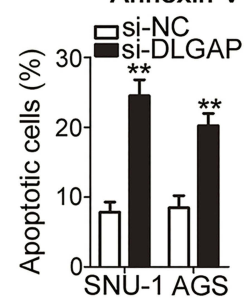

Figure 2 Depletion of DLGAPI-ASI expression inhibits the malignant process in SNU-I and AGS cells. (A) SNU-I and AGS cells were transfected with small interfering RNA (siRNA) for silencing DLGAPI-ASI expression (si-DLGAPI-ASI) or negative control siRNA (si-NC). The expression of DLGAPI-ASI was measured by RT-qPCR in the transfected cells. (B) The proliferative ability of SNU-I and AGS cells was assessed by CCK-8 assay after DLGAPI-ASI knockdown. (C) The proportion of apoptotic siDLGAPI-ASI-transfected or si-NC-transfected SNU-I and AGS cells was analyzed by flow cytometry (propidium iodide, PI). (D, E) Transwell migration and invasion assays were utilized to determine the migration and invasion of SNU-I and AGS cells in response to DLGAPI-ASI knockdown. *P $<0.05$ and $* * P<0.0 I$.

After miR-628-5p mimic was introduced into SNU-1 and AGS cells, miR-628-5p was remarkably upregulated compared with cells transfected with miR-NC (Figure 4A). CCK-8 assay and flow-cytometric analysis demonstrated that ectopic miR-628-5p expression resulted in a significant decrease in cell proliferation (Figure 4B) and increase in cell apoptosis (Figures 4C and D) in SNU-1 and AGS cells. Furthermore, transwell migration and invasion assays revealed that the migratory (Figure 4E) and invasive (Figure 4F) abilities of SNU-1 and AGS cells were greatly reduced after miR628-5p overexpression.

Identification of the direct targets of miR-628-5p is an essential step toward a better understanding of its participation in gastric carcinogenesis and GC progression. To elucidate the mechanism by which miR-628-5p suppressed GC progression, bioinformatics analysis was performed for miR628-5p target prediction. AEG-1 was selected for further analysis as it is known to be closely associated with the progression of $\mathrm{GC},{ }^{28-35,39}$ and the $3^{\prime}$-UTR of the AEG-1 mRNA was predicted to directly interact with miR-628-5p (Figure 4G). To test this hypothesis, the luciferase reporter assay was carried out to evaluate the direct interaction between miR-628-5p and the 3'-UTR of AEG-1. Transfection of the miR-628-5p mimic reduced the luciferase activity of the plasmid harboring the wild-type miR-628-5pbinding sites (1 and 2). By contrast, the luciferase activity barely changed in SNU-1 and AGS cells after cotransfection with the plasmid carrying the mutant AEG-1 3'-UTR (MutAEG-1) plus the miR-628-5p mimic (Figure 4H).

To further investigate the association between miR-6285p and $A E G-1$ in GC, RT-qPCR analysis was performed to measure $A E G-1$ expression in the 63 pairs of $\mathrm{GC}$ tissue samples and corresponding adjacent non-tumor tissue samples. The mRNA expression of $A E G-1$ was higher in the GC tissue samples than in the corresponding adjacent non-tumor tissues (Figure 4I). In addition, Spearman correlation analysis revealed an inverse correlation between the expression levels of miR-628-5p and $A E G-1$ mRNA among the GC tissue samples (Figure 4J; $r=-0.5365, \mathrm{P}<0.0001$ ). Furthermore, the mRNA (Figure 4K) and protein (Figure $4 \mathrm{~L}$ ) expression levels of AEG-1 were dramatically lower in SNU-1 and AGS cells overexpressing miR-628-5p, as evidenced by RT-qPCR 
A
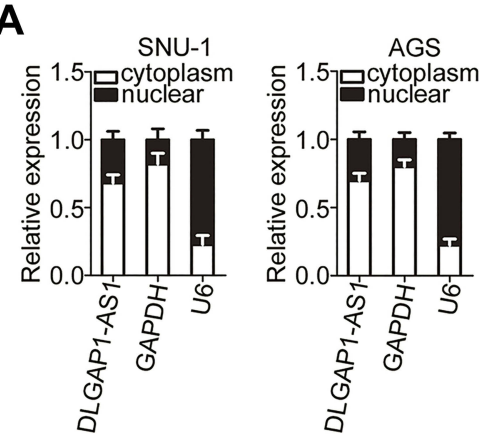

B

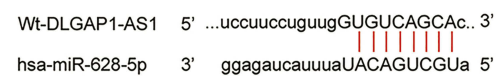

hsa-miR-628-5p 3' ggagaucauuuaUACAGUCGUa 5,

Mut-DLGAP1-AS1 5' ...uccuuccuguugGACAGUCGUc.. 3'

C
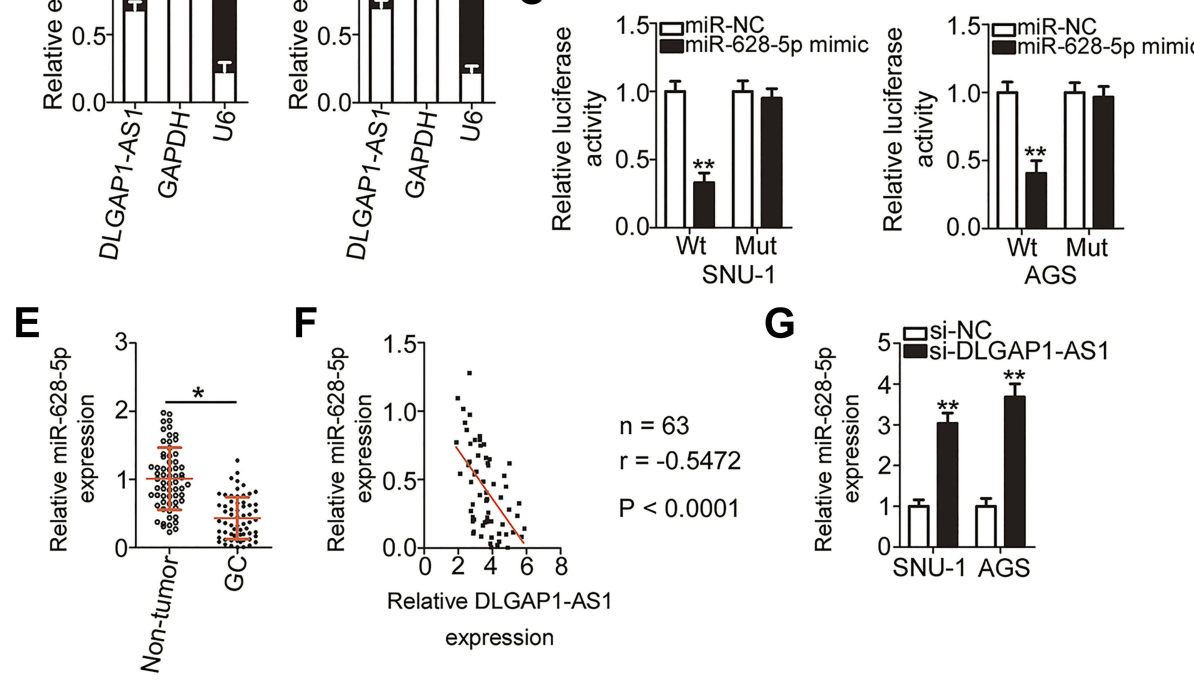

$\mathbf{F}$

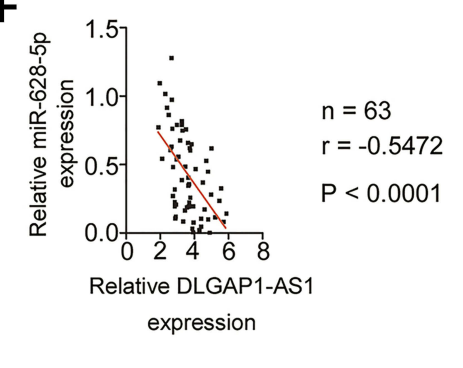

D

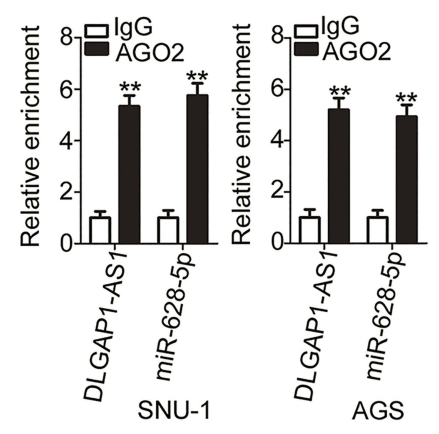

Figure 3 DLGAPI-ASI serves as a molecular sponge of miR-628-5p in gastric cancer (GC) cells. (A) Subcellular fraction extraction plus RT-qPCR analysis was performed to verify that DLGAPI-ASI was mainly present in the cytoplasm of SNU-I and AGS cells. (B) Bioinformatics prediction indicated the potential miR-628-5p binding site in the sequence of DLGAPI-ASI. The mutant binding sequences were also shown. (C) Luciferase activity was examined in SNU-I and AGS cells after cotransfection with wild-type (wt)-DLGAPI-ASI or mutant (Mut)-DLGAPI-ASI and miR-628-5p mimic or negative control miRNA mimic (miR-NC). (D) The interaction between DLGAPI-ASI and miR-628-5p in SNU-I and AGS cells was detected through RIP assay (Argonaute 2, AGO2). Immunoglobulin G (IgG) acted as the control. (E) MiR-628-5p expression in 63 pairs of GC tissue samples and the corresponding adjacent non-tumor tissue samples was analyzed by RT-qPCR. (F) Evaluation of the expression correlation between miR628-5p and DLGAPI-ASI in the 63 GC tissues by Spearman correlation analysis. $r=-0.5472, P<0.000$ I. (G) The expression level of miR-628-5P was measured by RTqPCR analysis in SNU-I and AGS cells after DLGAPI-ASI knockdown. $* \mathrm{P}<0.05$ and $* * \mathrm{P}<0.0 \mathrm{I}$.

and Western blotting. Collectively, these results clearly identified $A E G-1$ as a direct target gene of miR-628-5p in GC cells.

DLGAP1-AS1 functioned as a molecular sponge for miR-628-5p in GC cells, and AEG-1 functioned as a direct target of miR-628-5p. Accordingly, we further investigated whether DLGAP1-AS1 may influence the expression of AEG-1 in GC cells. The mRNA and protein levels of AEG-1 in SNU-1 and AGS cells after si-DLGAP1-AS1 or si-NC transfection were determined through RT-qPCR and Western blotting, respectively. As expected, depletion of DLGAP1-AS1 decreased the AEG-1 mRNA (Figure $4 \mathrm{M}$ ) and protein (Figure 4N) expression levels in SNU-1 and AGS cells. These results demonstrated that DLGAP1AS1 functioned as a ceRNA for miR-628-5p and consequently raised the expression of AEG-1 in GC cells.

\section{DLGAPI-ASI Functions Through the} Regulation of the miR-628-5p/AEG-I Axis in GC Cells

Rescue experiments were conducted to test the effectiveness of the DLGAP1-AS1/miR-628-5p/AEG-1 axis in GC cells. si-DLGAP1-AS1 was cotransfected with in-miR-628-5p or in-miR-NC into SNU-1 and AGS cells. RT-qPCR results verified that transfection with in-miR-628-5p significantly decreased the expression of miR-628-5p in SNU-1 and AGS cells (Figure 5A). The DLGAP1-AS1 knockdown-mediated decrease in AEG-1 mRNA (Figure 5B) and protein expression (Figure 5C) was reversed in SNU-1 and AGS cells after cotransfection with in-miR-628-5p. The proliferative capacity of SNU-1 and AGS cells was weakened by si-DLGAP1-AS1 relative to that of the si-NC group; interestingly, proliferative ability was partially regained following in-miR-628-5p cotransfection (Figure 5D). The induction of SNU-1 and AGS cell apoptosis due to DLGAP1-AS1 knockdown was reversed by in-miR $-628-5 p$ (Figures $5 E$ and F). Furthermore, the outcomes of transwell migration and invasion assays suggested that si-DLGAP1-AS1 transfection impaired SNU-1 and AGS cell migration (Figure 5G) and invasion (Figure $5 \mathrm{H})$. However, after cotransfecting in-miR-628-5p into both cells, the impacts caused by DLGAP1-AS1 silencing were largely recovered. 


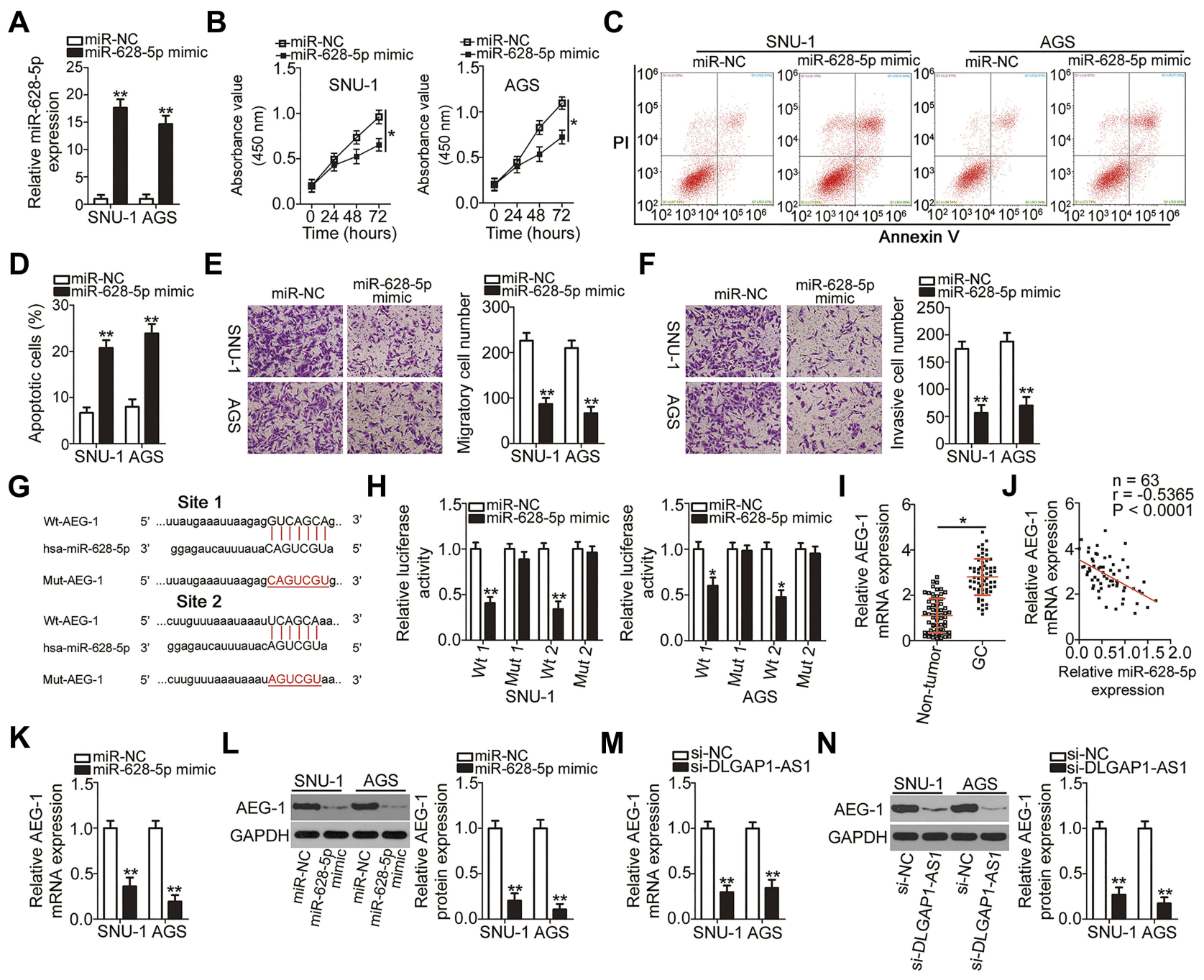

Figure 4 Astrocyte elevated gene I (AEG-I) is a direct target gene of miR-628-5p in gastric cancer (GC) cells and can be positively regulated by DLGAPI-ASI. (A) The expression of miR-628-5p in SNU-I and AGS cells was analyzed after transient transfection with either the miR-628-5p mimic or negative control miRNA mimic (miR-NC). (B-D) The proliferation and apoptosis of miR-628-5p-overexpressing SNU-I and AGS cells were measured by the CCK-8 assay and flow-cytometric analysis (propidium iodide, PI), respectively. (E, F) Transwell migration and invasion assays were conducted to assess the migration and invasiveness of SNU-I and AGS cells that were transfected with either the miR-628-5p mimic or miR-NC. (G) The predicted miR-628-5p-binding sequences in the 3'-UTR of AEG-I mRNA. Mutated sequences in the 3'UTR of AEG-I mRNA are highlighted in red. (H) Either wild-type (wt)-AEG-I or mutant (Mut)-AEG-I plus either the miR-628-5p mimic or miR-NC were transfected into SNU-I and AGS cells. Following $48 \mathrm{~h}$ of incubation, luciferase activity was determined using a dual-luciferase reporter assay system. (I) The relative expression of AEG-I mRNA in 63 pairs of GC tissue samples and corresponding adjacent non-tumor tissue samples was measured by RT-qPCR. (J) The expression correlation between miR-628$5 p$ and AEG-I mRNA in the 63 GC tissues was evaluated via Spearman correlation analysis. $r=-0.5365, P<0.000 I$. (K, L) The changes in AEG-I mRNA and protein expression in SNU-I and AGS cells after miR-628-5p overexpression were assessed via RT-qPCR and Western blotting. (M, N) RT-qPCR and Western blotting were conducted to measure the expression of AEG-I mRNA and protein expression in SNU-I and AGS cells after small interfering RNA (siRNA) for silencing DLGAPI-ASI expression (si-DLGAPI-ASI) or negative control siRNA (si-NC) transfection. *P $<0.05$ and **P $<0.01$.

The AEG-1 overexpression plasmid (pcDNA3.1-AEG1) or empty pcDNA3.1 plasmid was cotransfected with siDLGAP1-AS1 into SNU-1 and AGS cells. The efficiency of pcDNA3.1-AEG-1 was determined by Western blotting (Figure 6A). pcDNA3.1-AEG-1 or the empty pcDNA3.1 plasmid was transfected into DLGAP1-AS1 deficient-SNU -1 and AGS cells. Then, functional experiments were performed in these cells, and the results revealed that the recovery of AEG-1 expression abrogated the effects of DLGAP1-AS1 downregulation on the proliferation (Figure 6B), apoptosis (Figure 6C), migration (Figure 6D), and invasiveness (Figure 6E) of SNU-1 and AGS cells. The above results provided additional evidence that DLGAP1-AS1 worked as a ceRNA to facilitate the malignancy of GC cells at least partly by increasing the output of the miR-628-5p/AEG-1 axis. 

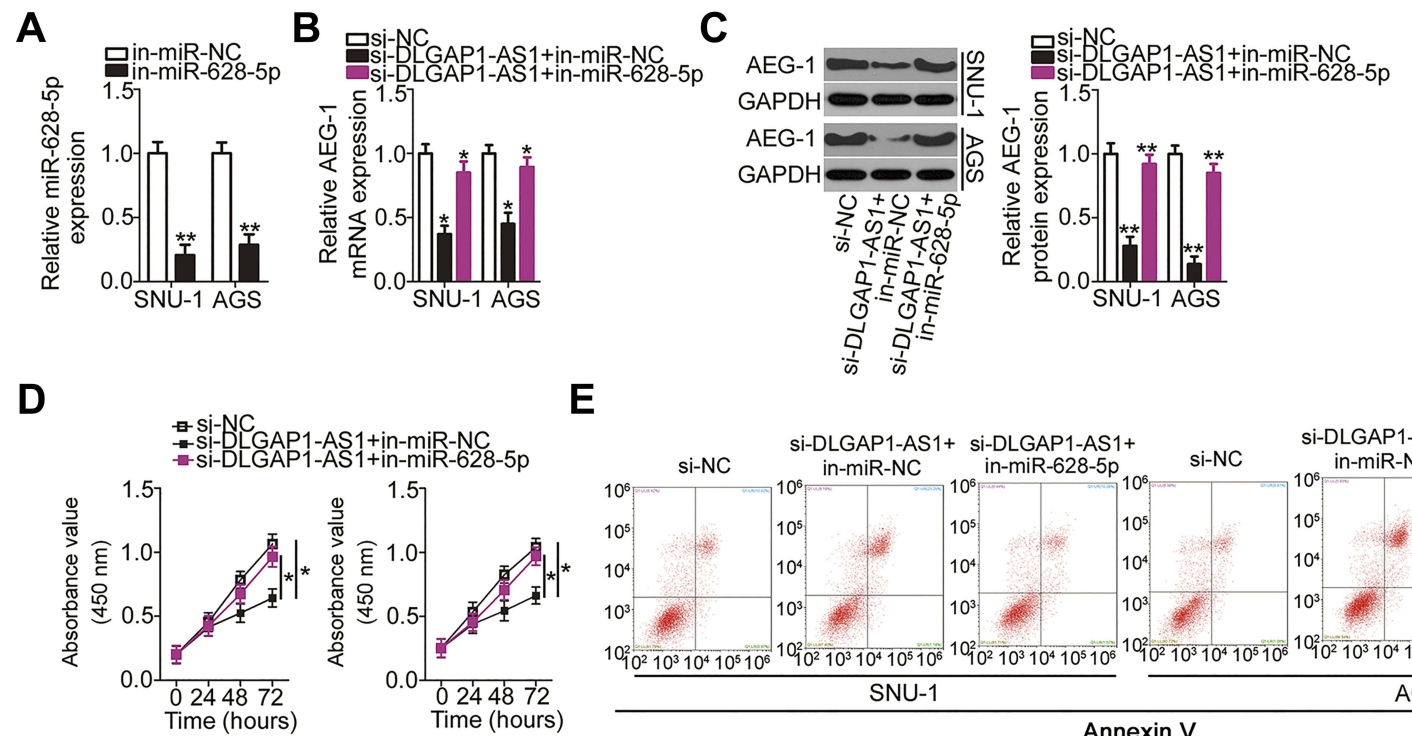

$\mathbf{F}$

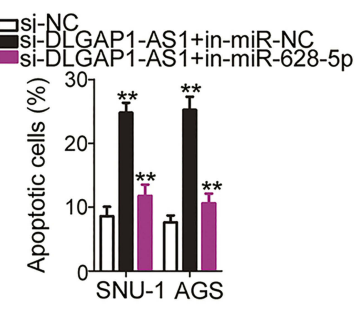

E

\section{.}

G

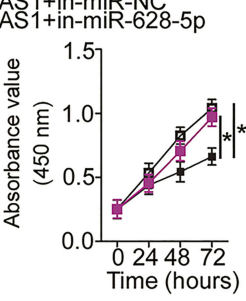

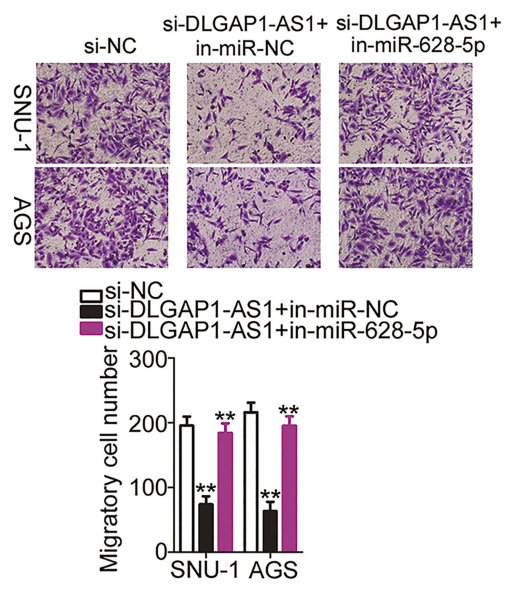

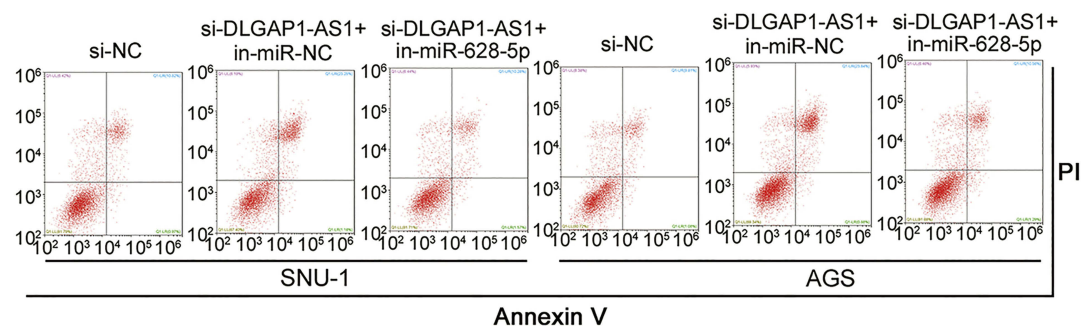

H

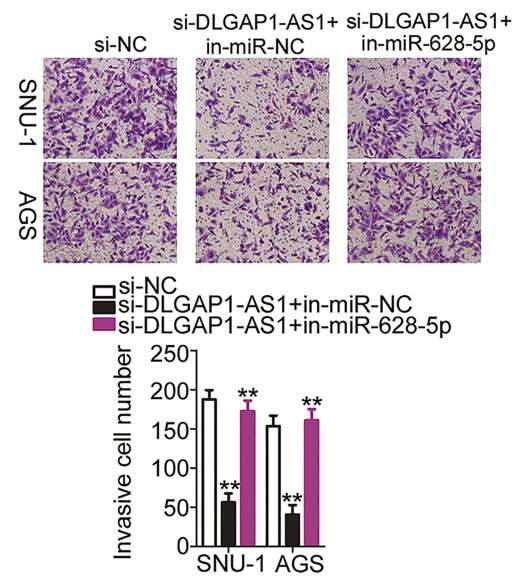

Figure 5 Inhibition of miR-628-5p abrogates the impacts of DLGAPI-ASI silencing in gastric cancer (GC) cells. (A) SNU-I and AGS cells were transfected with miR-628-5p inhibitor (in-miR-628-5p) or negative control miRNA inhibitor (in-miR-NC). After being transfected for 48 h, RT-qPCR was carried out to evaluate miR-628-5p expression. (B, C) In-miR-628-5p or in-miR-NC was transfected into SNU-I and AGS cells in the presence of small interfering RNA (siRNA) for silencing DLGAPI-ASI expression (siDLGAPI-ASI). Negative control siRNA (si-NC) was also transfected into SNU-I and AGS cells as the control. The expression levels of AEG-I mRNA and AEG-I protein were detected by RT-qPCR and Western blotting, respectively. (D-H) CCK-8 assay, flow-cytometric analysis (propidium iodide; PI), and transwell migration and invasion assays were respectively used for testing the proliferation, apoptosis, migration, and invasiveness of SNU-I and AGS cells treated as described above. *P $<0.05$ and $* * P<$ 0.01 .

\section{DLGAPI-ASI Knockdown Hinders GC}

\section{Tumor Growth in vivo}

To explore the effects of DLGAP1-AS1 on the tumor growth of GC cells in vivo, AGS cells stably transfected with either sh-DLGAP1-AS1 or sh-NC were inoculated into the flanks of nude mice to establish a transplanted tumor model. The tumor xenografts grew more slowly (Figures 7A and B), and the resultant tumor weight was significantly lower (Figure 7C) in the sh-DLGAP1-AS1 group than in the sh-NC group. Further analysis revealed that DLGAP1-AS1 was still decreased (Figure 7D) and miR-628-5p was increased (Figure 7E) in the tumor xenografts derived from DLGAP1-AS1-downregulated AGS cells. Furthermore, Western blotting showed that the amount of AEG-1 protein was decreased in the tumor xenografts obtained from the sh-DLGAP1-AS1 group (Figure 7F). These results suggest that the depletion of DLGAP1-AS expression inhibited the tumor growth of GC cells in vivo.

\section{Discussion}

Multiple lncRNAs have been found to be aberrantly expressed in GC, and this abnormal expression is strongly involved in the initiation and progression of GC. ${ }^{40-42}$ It is 


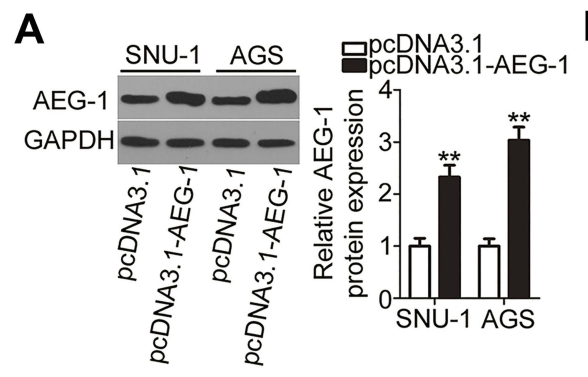

B

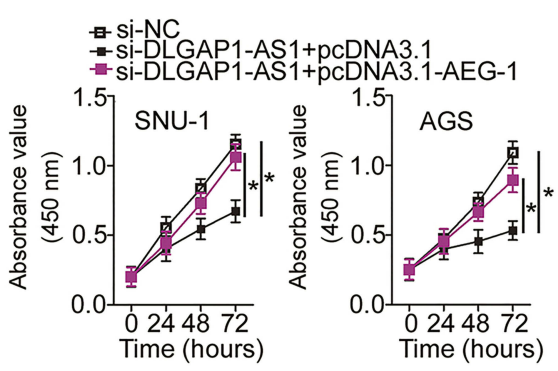

C

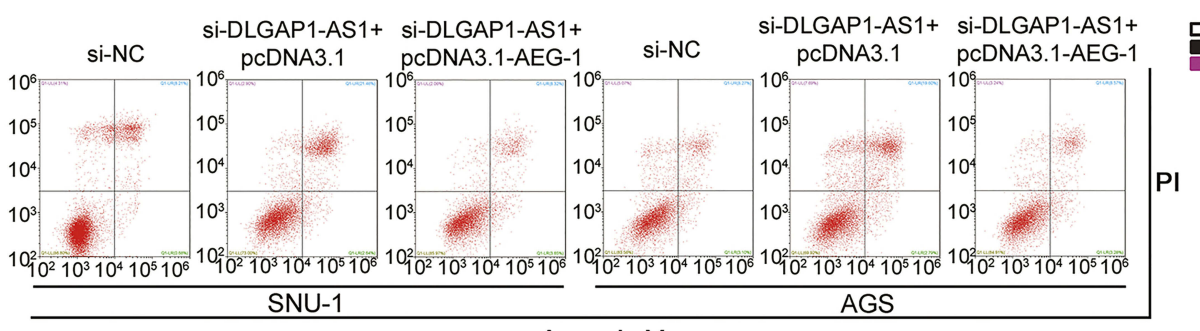

Annexin V

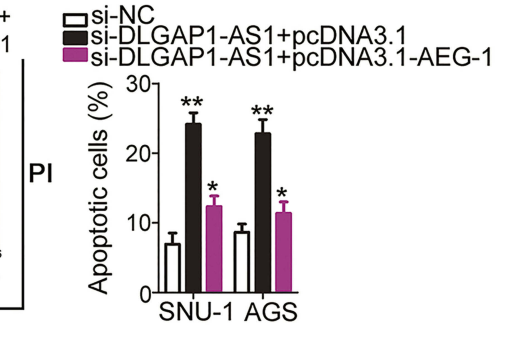

D

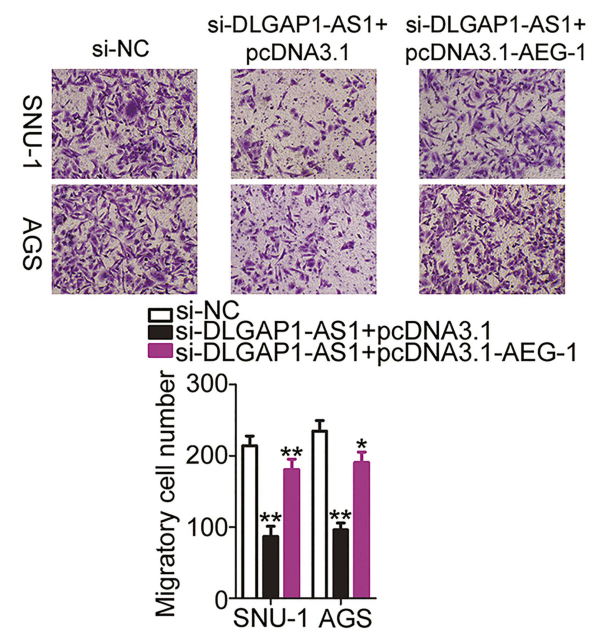

E

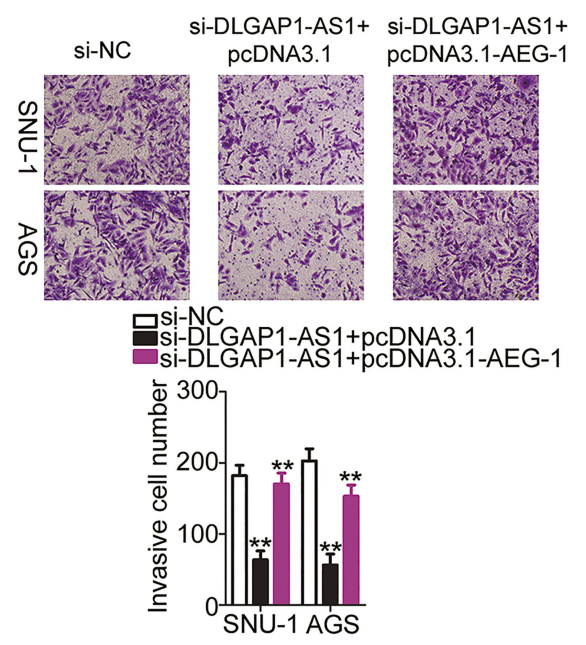

Figure 6 Restored astrocyte elevated gene I (AEG-I) expression reverses the effects of DLGAPI-ASI knockdown in gastric cancer (GC) cells. (A) SNU-I and AGS cells were transfected with the AEG-I overexpression plasmid pcDNA3.I-AEG-I or empty pcDNA3.I plasmid. Western blotting was conducted to evaluate AEG-I protein expression. (B-E) The pcDNA3.I-AEG-I was introduced into DLGAPI-ASI small interfering RNA (si-DLGAPI-ASI)-transfected SNU-I and AGS cells. Negative control siRNA (si-NC) was also transfected into SNU-I and AGS cells as the control. The proliferation, apoptosis, migration, and invasiveness of the aforementioned cells were investigated using CCK-8 assay, flow-cytometric analysis (propidium iodide; $\mathrm{PI}$ ), and transwell migration and invasion experiments, respectively. ${ }^{*} \mathrm{P}<0.05$ and $* * \mathrm{P}<0.0 \mathrm{I}$.

therefore important to explore the biological functions of dysregulated lncRNAs in GC as this may contribute to the development of effective therapeutic strategies for and improvement of the clinical outcomes of patients with GC. In this study, we evaluated DLGAP1-AS1 expression in GC and investigated the effects of DLGAP1-AS1 on the malignancy of GC in detail. To the best of our knowledge, this is the first study on the expression pattern and involvement of DLGAP1-AS1 in GC.

DLGAP1-AS1 expression is upregulated in hepatocellular carcinoma. ${ }^{23}$ DLGAP1-AS1 downregulation inhibits the proliferation and promotes the apoptosis of hepatocellular carcinoma. ${ }^{23}$ Nonetheless, whether DLGAP1-AS1 is deregulated in GC and, if so, whether its deregulation is closely related to the malignant characteristics of GC had not been elucidated. In this study, we demonstrated that DLGAP1-AS1 is overexpressed in GC tumors and cell lines. Increased DLGAP1-AS1 expression significantly correlated with tumor size, TNM stage, lymph node metastasis, and distant metastasis among our patients with GC. In addition, patients with GC and high DLGAP1-AS1 expression had shorter overall survival compared with 
A

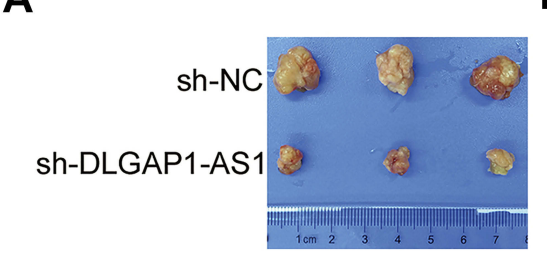

B

D

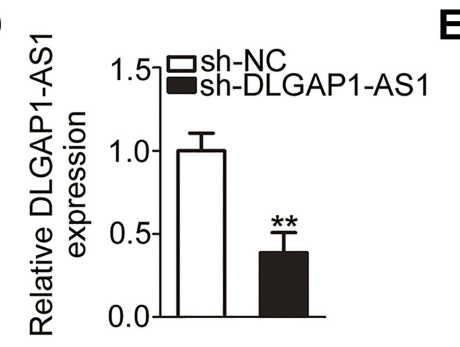

E

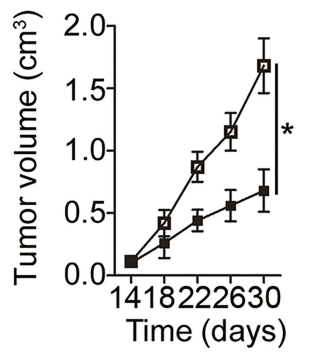

C

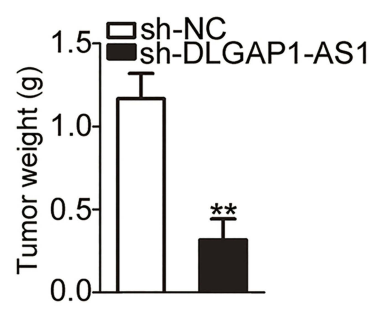

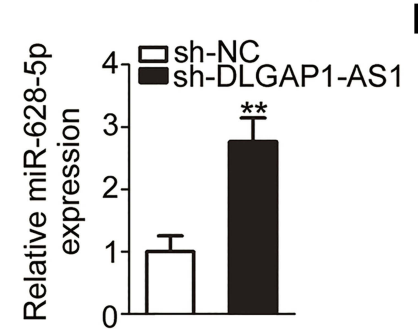

$F$

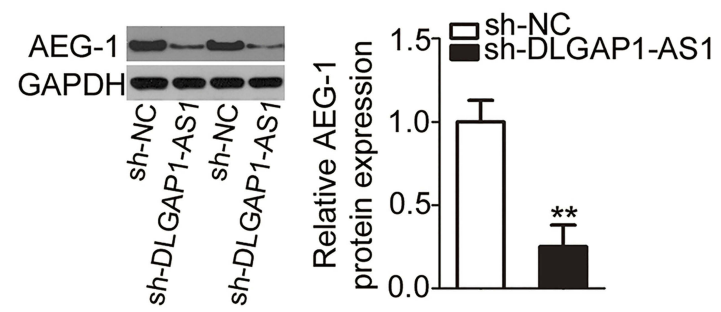

Figure 7 DLGAPI-ASI knockdown inhibits tumor growth in vivo. (A) Representative images of the tumor xenografts from groups DLGAPI-ASI short hairpin RNA (shRNA; sh-DLGAPI-ASI) and negative control shRNA (sh-NC). (B) The volume of tumor xenografts in groups sh-DLGAPI-ASI and sh-NC was measured every 4 days, and a growth curve was constructed accordingly. $(\mathbf{C})$ The tumor xenografts were excised at the end of the xenograft model experiment and were then weighed. (D-F) Total RNA and protein were extracted from the tumor xenografts and were then subjected to quantitation of DLGAPI-ASI, miR-628-5p, and Astrocyte elevated gene I (AEG-I) protein expression. $* \mathrm{P}<0.05$ and $* * \mathrm{P}<0.01$.

patients who had low DLGAP1-AS1 expression. Functionally, interference with DLGAP1-AS1 expression suppressed GC cell proliferation, migration, and invasion in vitro, as well as induced cell apoptosis and impaired tumor growth in vivo.

Investigation of the molecular mechanisms underlying the tumor-promoting effects of DLGAP1-AS1 in GC may help identify effective targets for anticancer therapies. As a factor considerably affecting post-transcriptional modulation, lncRNAs competitively decrease the binding of miRNAs to their target mRNAs by "sponge" adsorption, positively regulating the expression of oncogenic or tumor suppressive genes. ${ }^{43}$ Our bioinformatics prediction indicated that miR-628-5p has a putative DLGAP1-AS1 binding site. Further experimental validation found that DLGAP1-AS1 could directly interact with miR-628-5p in GC cells. In addition, miR-628-5p was weakly expressed in GC tissues and demonstrated a negative correlation with DLGAP1-AS1 expression. Furthermore, knockdown of DLGAP1-AS1 resulted in a notable increase of miR-628$5 \mathrm{p}$ expression in GC cells. After identifying AEG-1 as a target of miR-628-5p, we next investigated the regulatory relationship among DLGAP1-AS1, miR-628-5p and AEG-1. AEG-1 was positively regulated by DLGAP1AS1 in GC cells, and the regulatory influence was exerted through miR-628-5p sponging. In addition, rescue assays revealed that increasing the output of the miR-628-5p/
AEG-1 axis neutralized the DLGAP1-AS1 deficiencymediated GC progression inhibition. All in all, our study identified a ceRNA regulatory pathway in $\mathrm{GC}$ involving DLGAP1-AS1, miR-628-5p, and AEG-1.

Upregulation of miR-628-5p in osteosarcoma is correlated with poor clinical outcomes in patients. ${ }^{27} \mathrm{MiR}-628-$ $5 \mathrm{p}$ acts as an oncogenic miRNA in osteosarcoma and is involved in the control of cell proliferation, migration and invasion. ${ }^{27}$ On the contrary, miR-628-5p is downregulated in epithelial ovarian cancer $^{25}$ and glioma, ${ }^{26}$ and it performs anti-oncogenic roles in the progression of these malignancies. In this study, we first confirmed that miR628-5p expression is low in GC. Exogenous miR-628-5p expression played an inhibitory role in the aggressive behavior of GC cells.

$A E G-1$, also known as $M T D H$, is located in the chromosomal region $8 \mathrm{q} 22 .{ }^{44}$ AEG-1 is upregulated in a broad range of human malignant tumors, including thyroid carcinoma, ${ }^{45}$ bladder cancer, ${ }^{46}$ breast cancer, ${ }^{47}$ and glioma. ${ }^{48}$ AEG-1 is also overexpressed in $\mathrm{GC}$, and this overexpression correlates with the differentiation status, TNM stage, proliferative index (Ki-67), invasion depth, and lymph node metastasis among patients with GC. ${ }^{28-30}$ Patients with GC harboring high AEG1 expression demonstrate shorter overall survival than patients with low AEG-1 expression. ${ }^{30}$ In addition, multivariate analysis has identified AEG-1 as an independent prognostic factor for GC. ${ }^{30}$ AEG-1 is implicated in GC progression, including 
cell proliferation, cell cycle, apoptosis, metastasis, chemoresistance, and epithelial-mesenchymal transition. ${ }^{31-35}$ Herein, we demonstrated that DLGAP1-AS1, which harbors a miR$628-5 \mathrm{p}$-binding site, worked as a ceRNA to decrease the effective miR-628-5p amount and consequently raised the expression of AEG-1, thereby promoting the aggressive behavior of GC. Consequently, targeting the DLGAP1-AS1/miR628-5p/AEG-1 pathway might be an innovative modality for managing GC.

\section{Conclusions}

We herein, for the first time, report the significance of DLGAP1-AS1/miR-628-5p/AEG-1 pathway in the progression of GC. Mechanically, DLGAP1-AS1 facilitates the oncogenicity of GC cells through promoting miR-628-5p-regulated stability of AEG-1. Our findings suggest that the DLGAP1AS1/miR-628-5p/AEG-1 has substantial diagnostic, prognostic, and/or therapeutic potential for patients with GC.

\section{Ethical Approval and Informed Consent}

The experimental protocols used in the current study were approved by the Ethics Committee of Gaomi People's Hospital and were performed in accordance with the Declaration of Helsinki. In addition, all participants provided written informed consent prior to surgical resection. The animal experiments were approved by the Animal Ethical Committee of Gaomi People's Hospital. All experimental steps were performed in accordance with the Animal Protection Law of the People's Republic of China-2009 for experimental animals.

\section{Disclosure}

The authors report no conflicts of interest in this work.

\section{References}

1. Torre LA, Bray F, Siegel RL, Ferlay J, Lortet-Tieulent J, Jemal A. Global cancer statistics, 2012. CA Cancer J Clin. 2015;65(2):87-108. doi:10.3322/caac. 21262

2. Ferlay J, Shin HR, Bray F, Forman D, Mathers C, Parkin DM. Estimates of worldwide burden of cancer in 2008: GLOBOCAN 2008. Int J Cancer. 2010;127(12):2893-2917. doi:10.1002/ijc.25516

3. Thiel A, Ristimaki A. Targeted therapy in gastric cancer. APMIS. 2015;123(5):365-372. doi:10.1111/apm.12359

4. Moon YW, Jeung HC, Rha SY, et al. Changing patterns of prognosticators during 15-year follow-up of advanced gastric cancer after radical gastrectomy and adjuvant chemotherapy: a 15-year follow-up study at a single korean institute. Ann Surg Oncol. 2007;14 (10):2730-2737. doi:10.1245/s10434-007-9479-4
5. Cunningham D, Allum WH, Stenning SP, et al. Perioperative chemotherapy versus surgery alone for resectable gastroesophageal cancer. NEngl JMed. 2006;355(1):11-20. doi:10.1056/NEJMoa055531

6. Duarte HO, Gomes J, Machado JC, Reis CA. Gastric cancer: basic aspects. Helicobacter. 2018;23(Suppl 1):e12523. doi:10.1111/ hel. 12523

7. Sun F, Lu X, Li H, et al. Special AT-rich sequence binding protein 1 regulates the multidrug resistance and invasion of human gastric cancer cells. Oncol Lett. 2012;4(1):156-162. doi:10.3892/ol.2012.681

8. Murugan AK, Munirajan AK, Alzahrani AS. Long noncoding RNAs: emerging players in thyroid cancer pathogenesis. Endocr Relat Cancer. 2018;25(2):R59-R82. doi:10.1530/ERC-17-0188

9. Xin Y, Li Z, Shen J, Chan MT, Wu WK. CCAT1: a pivotal oncogenic long non-coding RNA in human cancers. Cell Prolif. 2016;49 (3):255-260. doi:10.1111/cpr.12252

10. Yu Y, Yang J, Li Q, Xu B, Lian Y, Miao L. LINC00152: a pivotal oncogenic long non-coding RNA in human cancers. Cell Prolif. 2017;50(4). doi:10.1111/cpr.12368

11. Zhao J, Zhang C, Gao Z, Wu H, Gu R, Jiang R. Long non-coding RNA ASBEL promotes osteosarcoma cell proliferation, migration, and invasion by regulating microRNA-21. J Cell Biochem. 2018;119 (8):6461-6469. doi:10.1002/jcb.26671

12. Ghafouri-Fard S, Taheri M. Long non-coding RNA signature in gastric cancer. Exp Mol Pathol. 2019;104365.

13. Ren W, Zhang J, Li W, et al. A tumor-specific prognostic long non-coding RNA signature in gastric cancer. Med Sci Monit. 2016;22:3647-3657. doi:10.12659/MSM.901190

14. Zhu X, Tian X, Yu C, et al. A long non-coding RNA signature to improve prognosis prediction of gastric cancer. Mol Cancer. 2016;15 (1):60. doi:10.1186/s12943-016-0544-0

15. Yang H, Peng M, Li Y, Zhu R, Li X, Qian Z. LINC00703 acts as a tumor suppressor via regulating miR-181a/KLF6 axis in gastric cancer. J Gastric Cancer. 2019;19(4):460-472. doi:10.5230/ jgc.2019.19.e43

16. Shao G, Zhao Z, Zhao W, et al. Long non-coding RNA MALAT1 activates autophagy and promotes cell proliferation by downregulating microRNA-204 expression in gastric cancer. Oncol Lett. 2020;19 (1):805-812. doi:10.3892/ol.2019.11184

17. Xie C, Guo Y, Lou S. LncRNA ANCR promotes invasion and migration of gastric cancer by regulating FoxO1 expression to inhibit macrophage M1 polarization. Dig Dis Sci. 2020. doi:10.1007/s10620019-06019-1

18. Han Y, Wu N, Jiang M, et al. Long non-coding RNA MYOSLID functions as a competing endogenous RNA to regulate MCL-1 expression by sponging miR-29c-3p in gastric cancer. Cell Prolif. 2019;52(6):e12678. doi:10.1111/cpr.12678

19. Zhou C, Zhao J, Liu J, et al. LncRNA SNHG16 promotes epithelialmesenchymal transition via down-regulation of DKK3 in gastric cancer. Cancer Biomark. 2019;26(4):393-401. doi:10.3233/CBM190497

20. Kojima S, Goto Y, Naya Y. The roles of microRNAs in the progression of castration-resistant prostate cancer. J Hum Genet. 2017;62 (1):25-31. doi:10.1038/jhg.2016.69

21. Bartel DP. MicroRNAs: genomics, biogenesis, mechanism, and function. Cell. 2004;116(2):281-297. doi:10.1016/S0092-8674(04) 00045-5

22. Mendell JT, Olson EN. MicroRNAs in stress signaling and human disease. Cell. 2012;148(6):1172-1187. doi:10.1016/j.cell.2012.02.005

23. Peng X, Wei F, Hu X. Long noncoding RNA DLGAP1-AS1 promotes cell proliferation in hepatocellular carcinoma via sequestering miR-486-5p. J Cell Biochem. 2020;121(2):1953-1962. doi:10.1002/ jcb. 29430

24. Zhou L, Jiao X, Peng X, Yao X, Liu L, Zhang L. MicroRNA-628-5p inhibits invasion and migration of human pancreatic ductal adenocarcinoma via suppression of the AKT/NF-kappa B pathway. $J$ Cell Physiol. 2020. doi:10.1002/jcp.29468 
25. Li M, Qian Z, Ma X, et al. MiR-628-5p decreases the tumorigenicity of epithelial ovarian cancer cells by targeting at FGFR2. Biochem Biophys Res Commun. 2018;495(2):2085-2091. doi:10.1016/j. bbrc.2017.12.049

26. Xie P, Wang Y, Liao Y, et al. MicroRNA-628-5p inhibits cell proliferation in glioma by targeting DDX59. J Cell Biochem. 2019;120 (10):17293-17302. doi:10.1002/jcb.28991

27. Wang JY, Lu SB, Wang JQ. miR-628-5p promotes the growth and migration of osteosarcoma by targeting IFI44L. Biochem Cell Biol. 2019;999:1-7.

28. Luo Y, Zhang X, Tan Z, et al. Astrocyte elevated gene-1 as a novel clinicopathological and prognostic biomarker for gastrointestinal cancers: a meta-analysis with 2999 patients. PLoS One. 2015;10(12): e0145659. doi:10.1371/journal.pone.0145659

29. Dong L, Qin S, Li Y, et al. High expression of astrocyte elevated gene-1 is associated with clinical staging, metastasis, and unfavorable prognosis in gastric carcinoma. Tumour Biol. 2015;36(3):2169-2178. doi:10.1007/s13277-014-2827-7

30. Jian-bo X, Hui W, Yu-long H, et al. Astrocyte-elevated gene-1 overexpression is associated with poor prognosis in gastric cancer. Med Oncol. 2011;28(2):455-462. doi:10.1007/s12032-010-9475-6

31. Jiao Y, Yang H, Qian J, et al. miR36645P suppresses the proliferation and metastasis of gastric cancer by attenuating the NFkappaB signaling pathway through targeting MTDH. Int $J$ Oncol. 2019;54 (3):845-858. doi:10.3892/ijo.2019.4680

32. Wang F. miR-384 targets metadherin gene to suppress growth, migration, and invasion of gastric cancer cells. J Int Med Res. 2019;47 (2):926-935. doi:10.1177/0300060518817171

33. Liao Z, Li Y, Zhou Y, Huang Q, Dong J. MicroRNA-197 inhibits gastric cancer progression by directly targeting metadherin. Mol Med Rep. 2018;17(1):602-611. doi:10.3892/mmr.2017.7908

34. Wu S, Yang L, Wu D, et al. AEG-1 induces gastric cancer metastasis by upregulation of eIF4E expression. J Cell Mol Med. 2017;21 (12):3481-3493. doi:10.1111/jcmm. 13258

35. Du Y, Jiang B, Song S, et al. Metadherin regulates actin cytoskeletal remodeling and enhances human gastric cancer metastasis via epithelial-mesenchymal transition. Int J Oncol. 2017;51(1):63-74. doi:10.3892/ijo.2017.4002

36. Dong BS, Shi MJ, Su SB, Zhang H. Insight into long noncoding competing endogenous RNA networks in hepatic fibrosis: the potential implications for mechanism and therapy. Gene. 2019;687:255-260. doi:10.1016/j.gene.2018.11.063
37. Hao NB, He YF, Li XQ, Wang K, Wang RL. The role of miRNA and lncRNA in gastric cancer. Oncotarget. 2017;8(46):81572-81582. doi:10.18632/oncotarget.19197

38. Wang L, Cho KB, Li Y, Tao G, Xie Z, Guo B. Long noncoding RNA (lncRNA)-mediated competing endogenous RNA networks provide novel potential biomarkers and therapeutic targets for colorectal cancer. Int J Mol Cell Med. 2019;20(22):5758.

39. Huang Y, Li LP. Progress of cancer research on astrocyte elevated gene-1/Metadherin (review). Oncol Lett. 2014;8(2):493-501. doi:10.3892/ol.2014.2231

40. Tam C, Wong JH, Tsui SKW, Zuo T, Chan TF, Ng TB. LncRNAs with miRNAs in regulation of gastric, liver, and colorectal cancers: updates in recent years. Appl Microbiol Biotechnol. 2019;103 (12):4649-4677.

41. Fanelli GN, Gasparini P, Coati I, et al. LONG-NONCODING RNAs in gastroesophageal cancers. Noncoding RNA Res. 2018;3 (4):195-212. doi:10.1016/j.ncrna.2018.10.001

42. Virgilio E, Giarnieri E, Giovagnoli MR, et al. Long non-coding RNAs in the gastric juice of gastric cancer patients. Pathol Res Pract. 2018;214(9):1239-1246. doi:10.1016/j.prp.2018.07.023

43. Ye Y, Shen A, Liu A. Long non-coding RNA H19 and cancer: a competing endogenous RNA. Bull Cancer. 2019;106 (12):1152-1159. doi:10.1016/j.bulcan.2019.08.011

44. Anttila V, Stefansson H, Kallela M, et al. Genome-wide association study of migraine implicates a common susceptibility variant on 8q22.1. Nat Genet. 2010;42(10):869-873.

45. Li WF, Wang G, Zhao ZB, Liu CA. High expression of metadherin correlates with malignant pathological features and poor prognostic significance in papillary thyroid carcinoma. Clin Endocrinol (Oxf). 2015;83(4):572-580. doi:10.1111/cen.12683

46. Nikpour M, Emadi-Baygi M, Fischer U, Niegisch G, Schulz WA, Nikpour P. MTDH/AEG-1 contributes to central features of the neoplastic phenotype in bladder cancer. Urol Oncol. 2014;32 (5):670-677. doi:10.1016/j.urolonc.2013.11.005

47. Li J, Zhang N, Song LB, et al. Astrocyte elevated gene-1 is a novel prognostic marker for breast cancer progression and overall patient survival. Clin Cancer Res. 2008;14(11):3319-3326. doi:10.1158/ 1078-0432.CCR-07-4054

48. Tong L, Chu M, Yan B, et al. MTDH promotes glioma invasion through regulating miR-130b-ceRNAs. Oncotarget. 2017;8 (11):17738-17749. doi:10.18632/oncotarget.14717

\section{Publish your work in this journal}

Cancer Management and Research is an international, peer-reviewed open access journal focusing on cancer research and the optimal use of preventative and integrated treatment interventions to achieve improved outcomes, enhanced survival and quality of life for the cancer patient.
The manuscript management system is completely online and includes a very quick and fair peer-review system, which is all easy to use. Visit http://www.dovepress.com/testimonials.php to read real quotes from published authors. 